\title{
Micropatterning of Aptamer Beacons to Create Cytokine-Sensing Surfaces
}

\author{
Nazgul Tuleuova ${ }^{1,2}$ and Alexander Revzin ${ }^{1}$ \\ ${ }^{1}$ Department of Biomedical Engineering, University of California, Davis, 451 Health Sciences Drive, \#2519, Davis, \\ CA 95616, USA; and ${ }^{2}$ National Center for Biotechnology, Astana, Kazakhstan
}

(Received 16 September 2010; accepted 8 November 2010; published online 20 November 2010)

Associate Editor David J. Odde oversaw the review of this article.

\begin{abstract}
Aptamer beacons are DNA or RNA probes that bind proteins or small molecules of interest and emit signal directly upon interaction with the target analyte. This paper describes micropatterning of aptamer beacons for detection of IFN- $\gamma$ - an important inflammatory cytokine. The beacon consisted of a fluorophore-labeled aptamer strand hybridized with a shorter, quencher-carrying complementary strand. Cytokine molecules were expected to displace quenching strands of the beacon, disrupting FRET effect and resulting in fluorescence signal. The glass substrate was first micropatterned with poly(ethylene glycol) (PEG) hydrogel microwells (35 $\mu \mathrm{m}$ diameter individual wells) so as to define sites for attachment of beacon molecules. PEG microwell arrays were then incubated with avidin followed by biotin-aptamerfluorophore constructs. Subsequent incubation with quencher-carrying complementary strands resulted in formation of DNA duplex and caused quenching of fluorescence due to FRET effect. When exposed to IFN- $\gamma$, microwells changed fluorescence from low (quencher hybridized with fluorophore-carrying strand) to high (quenching strand displaced by cytokine molecules). The fluorescence signal was confined to microwells, was changing in real-time and was dependent on the concentration of IFN- $\gamma$. In the future, we plan to co-localize aptamer beacons and cells on micropatterned surfaces in order to monitor in real-time cytokine secretion from immune cells in microwells.
\end{abstract}

Keywords-High-throughput screening, Biosensors, Cell arrays, Aptamers, Surface micropatterning.

\section{INTRODUCTION}

High-throughput screening of cells has become an important part of drug discovery, cancer research, immune cell analysis and toxicology studies. ${ }^{1,13,14,30}$ The desire to increase throughput is reflected in

Address correspondence to Alexander Revzin, Department of Biomedical Engineering, University of California, Davis, 451 Health Sciences Drive, \#2519, Davis, CA 95616, USA. Electronic mail: arevzin@ucdavis.edu evolution of a commercial microtiter plate from 96 to 384 to 1536 well format. In addition to increased information content, high-throughput technologies may also offer a better understanding of cellular heterogeneity. In this regard, microfabrication approaches are particularly advantageous for decreasing the size of the microwells to enable capture and culture of single cells. $4,6,18,20,21,29,31$ These single cell arrays may be thought of as providing both the ultimate throughput and the most nuanced view of heterogeneity within a given cell population.

An important question that remains to be addressed is how to analyze function of cells on micropatterned surfaces? If one is interested in analysis of heterogeneity on cell-by-cell basis then techniques relying on cells secreting into common media (e.g. ELISA) become unsuitable. At the present time, the analysis of cell function within arrays or on micropatterned surfaces is largely limited to intracellular markers, ${ }^{10,22,27,31}$ however, it is advantageous to be able to monitor secreted products in cell arrays.

Immunology is one area where analysis of secreted products is important. Immune cells are highly specialized and heterogeneous, with phenotype being frequently defined by the types of cytokines these cells produce. For example, secretion of IFN- $\gamma-\mathrm{a} 17 \mathrm{kDa}$ protein-is used to subcategorize CD4 T-cells into T-helper 1 (Th1) cells $\left(\mathrm{IFN}-\gamma^{+}\right)$vs. Th2 cells $\left(\mathrm{IFN}-\gamma^{-}\right) \cdot{ }^{15}$ Production of IFN- $\gamma$ in T-cells is associated with vigorous immune response to infections such as HIV and therefore represents an important correlate of immune cell function. ${ }^{16,17}$ Love and co-workers as well as our group have been developing micropatterned surfaces that enable detection of proteins secreted by the immune cells to permit high-throughput analysis of immune cell function. ${ }^{2,9,21,24,25,32,33}$

An approach pursued by our lab has relied on fabrication of poly(ethylene glycol) (PEG) hydrogel 
microwells on glass and immobilization of monoclonal antibodies (Abs) for capturing single immune cells within the wells. ${ }^{21}$ A more recent variant of this approach was to co-immobilize Abs specific for T-cells and IFN- $\gamma$ to be able to capture single CD4 T-cells and to detect secreted IFN- $\gamma$ in the same microwells. ${ }^{33}$ This approach allowed to assign cytokine production to specific cells within the array, however, Ab-based sandwich immunoassay employed for detection of IFN- $\gamma$ provided only an end-point measurement, revealing no temporal information about cytokine release from cells.

DNA or RNA aptamers have emerged as an alternative to Abs for affinity-based biosensors. ${ }^{7}$ Advantages of aptamers include low cost and chemical stability, but arguably their biggest advantage over Abs is the possibility of engineering beacons that emit signal directly upon analyte binding. ${ }^{8,11,26}$ Recently, our group has developed fluorescence resonance energy transfer (FRET)-based aptamer beacon for detection of IFN $-\gamma .{ }^{28}$ In the present study, IFN- $\gamma$ aptamer beacons were immobilized inside microwell arrays to create cytokine-sensing micropatterned surfaces. As shown in Fig. 1 beacons consisted of fluorophorelabeled DNA aptamer hybridized to a complementary quenching strand and attached inside hydrogels via avidin-biotin binding. In the absence of analyte, no signal was observed because of the FRET effect; however, exposure of microwells to IFN- $\gamma$ resulted in displacement of the quenching strand and appearance of fluorescence. Importantly, fluorescence signal was dependent on the analyte concentration, was confined to the microwells and was changing as a function of time. This study represents a step towards development of sensing micropatterned surfaces that may be used to both organize cells into high-density arrays and to detect secreted products in real-time on cell-by-cell or well-by-well basis. We envision IFN- $\gamma$ sensing micropatterned surfaces as having immediate impact for the analysis of antigen-specific CD4 and CD8 T-cells.

\section{MATERIALS AND METHODS}

\section{Chemicals and Materials}

Glass slides $\left(75 \times 25 \mathrm{~mm}^{2}\right)$ were obtained from VWR (West Chester, PA). 3-(3-acryloxypropyl) trichlorosilane was purchased from Gelest, Inc. (Morrisville, PA). Anhydrous toluene (99.9\%), 2-hydroxy-2methylpropiophenone (photoinitiator), poly (ethylene glycol) diacrylate (PEG-DA, MW 575), HEPES, KCl, EDTA, $\mathrm{MgCl} 2$, were obtained from Sigma-Aldrich (St. Louis, MO). Neutravidin was purchased from Invitrogen (Carlsbad, CA). Recombinant human IFN- $\gamma$ from R\&D systems (Minneapolis, MN). The following buffers were used in this study: HKE buffer (10 mM Hepes, $100 \mathrm{mM} \mathrm{KCl,} 1 \mathrm{mM}$ EDTA, pH 7.4), HKMT washing buffer (10 mM Hepes, $100 \mathrm{mM} \mathrm{KCl}$, $1 \mathrm{mM} \mathrm{MgCl} 2,0.05 \%$ Tween20, $\mathrm{pH}$ 7.4). 3' biotinylated IFN- $\gamma$ aptamer carrying Alexa 488 label (FA) and 3'BHQ-1-labeled complementary oligo (Q) were synthesized by IDT Technologies (San Diego, CA). Oligonucleotide sequences and modifications used in this study are as follows:

\section{Aptamer-5'-6-FA- T GGG GTT GGT TGT GTT GGG TGT TGT GT-Biotin-3'}

Quenching strand-5'-ACAACCAACCCCA-BHQ-1-3'

Prior to their use, oligonucleotides were heated at $95{ }^{\circ} \mathrm{C}$ for $3 \mathrm{~min}$ and then allowed to cool slowly to room temperature. Oligonucleotide samples were kept overnight at $4{ }^{\circ} \mathrm{C}$ until their use.

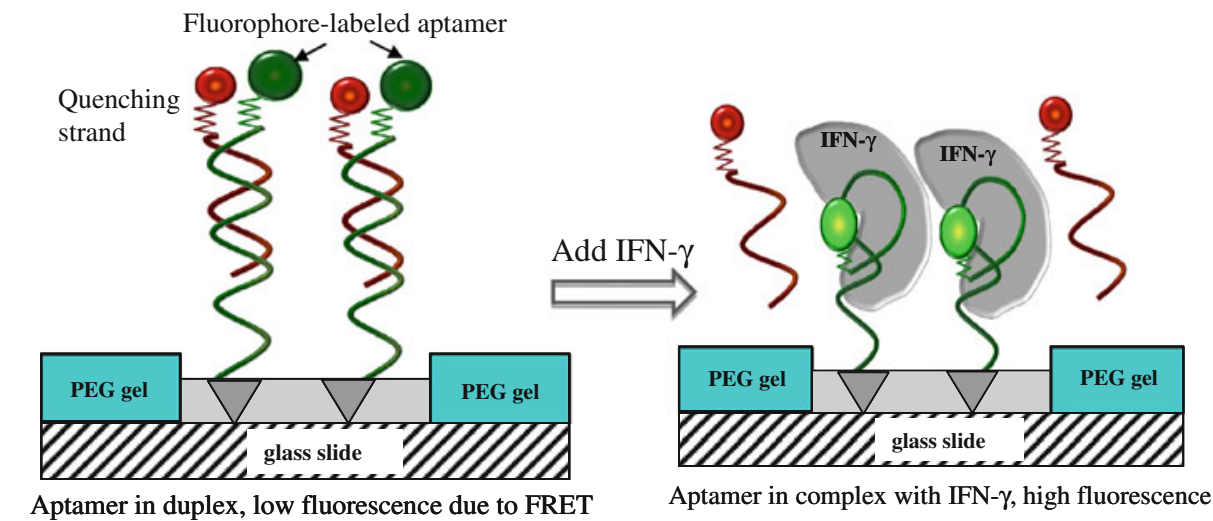

FIGURE 1. Schematic description of cytokine-sensing microwells. Aptamer beacons were immobilized inside micorwells using avidin-biotin binding. A beacon molecule consisted of fluorophore-labeled DNA aptamer and a quencher-labeled complementary strand. In duplex, fluorescence of an aptamer was quenched due to FRET effect resulting from proximity of fluorophore-labeled aptamer to acceptor-carrying complementary strand. Binding of IFN- $\gamma$ disrupted DNA duplex and resulted in fluorescence signal. 


\section{Fabrication of PEG Hydrogel Microwells}

Prior to PEG gel immobilization, the glass substrates were modified using a silane coupling agent. The substrates were exposed to $\mathrm{O}_{2}$ plasma for $3 \mathrm{~min}$ at $300 \mathrm{~W}$, placed into $\mathrm{N}_{2}$ filled glove bag and immersed in $2 \mathrm{mM}$ solution of (3-acryloxypropyl) trichlorosilane in toluene. The silane self-assembly was allowed to proceed for $1 \mathrm{~h}$ under $\mathrm{N}_{2}$ purge, after which the substrates were removed, rinsed in toluene and dried using $\mathrm{N}_{2}$ gas. The substrates were then placed in an oven for $3 \mathrm{~h}$ at $100{ }^{\circ} \mathrm{C}$ to cure the silane layer. This silanization procedure has been used by us previously for anchoring of the PEG hydrogel microstructures to glass substrates. Figure 2 illustrates the process flow for the fabrication of PEG hydrogel micropatterns. PEG hydrogel patterns were fabricated from the precursor solution of PEG-DA (MW 575) and 2\% (w/v) photoinitiator, DMPA. This prepolymer solution was spin-coated at $800 \mathrm{rpm}$ for $4 \mathrm{~s}$ onto a standard $3 \times 1 \mathrm{in}$. glass slide and then exposed to UV light at $65 \mathrm{~mW} / \mathrm{cm}^{2}$ for $15 \mathrm{~s}$ to convert liquid prepolymer into cross-linked hydrogel. Unpolymerized PEG solution was removed by development in DI water for $5 \mathrm{~min}$. This process resulted in formation of $35 \mu \mathrm{m}$ diameter PEG hydrogel wells on glass.

\section{Immobilization of Aptamer Beacons Inside Hydrogel Microwells}

The glass slides with micropatterned PEG hydrogel wells were incubated with $1 \mathrm{mg} / \mathrm{mL}$ neutroavidin in HKE buffer solution overnight at $4{ }^{\circ} \mathrm{C}$. Subsequently, surfaces were washed with HKE with $0.05 \%$ Tween and then immersed in $1 \mu \mathrm{M}$ solution of biotinylated aptamer-Alexa488 in HKE for $1 \mathrm{~h}$ at $37^{\circ} \mathrm{C}$. To visualize protein immobilization, neutravidin was replaced with Alexa546-labeled avidin and imaged using Nikon Eclipse LV100 inverted microsope (Nikon, Japan).

\section{Integration of Sensing Microwells with Microfluidics and Detection of IFN- $\gamma$}

PDMS microfluidic devices were fabricated using standard SU-8 processing and soft lithography protocols. The design of the microfluidic devices used in these experiments has been described in our previous publications. ${ }^{32,33}$ Briefly, the microfluidic device contained two flow chambers with width-length-height dimensions of $3 \times 10 \times 0.1 \mathrm{~mm}$ and a network of independently addressed auxiliary channels. The auxiliary channels were used to apply negative pressure (vacuum suction) to the PDMS mold and to reversibly secure it on top of a glass substrate. This strategy allowed to seal a fluid conduit on top of the glass slide without compromising integrity of PEG hydrogel arrays. The inlet/outlet holes were punched with a blunt 16 gauge needle. A $5 \mathrm{~mL}$ syringe was connected to silicone tubing (1/32 inch I.D., Fisher), which was attached to the outlet of the flow chamber with a metal insert cut from a 20 gauge needle. A blunt, shortened 20 gauge needle carrying a plastic hub was inserted in the inlet. A pressure-driven flow in the microdevice was created by withdrawing the syringe positioned at theoutlet with a precision syringe pump (Harvard Apparatus, Boston, MA).

PDMS microchannel device was aligned on the glass slide to fit PEG microwell patterns. Hydrogel microwells were incubated with avidin and biotinylated aptamer-fluorophore prior to integration with fluidic channels. After priming with HKE buffer, quencherlabeled antisense oligonucleotide was injected into microfluidic channels at concentration of $10 \mu \mathrm{M}$ and left to hybridize with aptamer for $30 \mathrm{~min}$ at room temperature. This step resulted in immobilization of an aptamer-fluorophore/antisense-quencher duplex on the surface of the microfluidic channels.

During cytokine detection experiments, IFN- $\gamma$ was injected into the microfluidic device at concentrations ranging from 1.25 to $100 \mathrm{nM}$ in HKE buffer. The change in fluorescence due to cytokine-aptamer beacon interactions was monitored using a Zeiss $200 \mathrm{M}$ epifluorescence microscope (Carl Zeiss MicroImaging, Inc. Thornwood, NY) equipped with xioCam MRm (CCD monochrome, 1300 pixels $\times 1030$ pixels). Objectives, camera and fluorescence filters were computer controlled through a PCI interface. Image acquisition and fluorescence analysis were carried out using AxioVision software (Carl Zeiss MicroImaging, Inc. Thornwood, NY).

\section{RESULTS AND DISCUSSION}

This paper describes the development of IFN- $\gamma$ sensing micropatterned surfaces. Glass substrates were micropatterned with arrays of PEG hydrogel microwells and were modified with aptamer beacons via avidin-biotin coupling chemistry. The fluorescence signal emitted by the microwell arrays was directly proportional to concentration of IFN- $\gamma$. In the future, these novel cytokine sensing microwells will be used to arrange immune cells into high-density arrays and determine cytokine secretion on cell-by-cell basis.

\section{Fabricating PEG Hydrogel Micropatterns on Glass}

Glass substrates were micropatterned with PEG hydrogels to define sites for binding of aptamer beacons. Hydrogel micropatterning followed the process presented in Fig. 2, with glass slides silanized to 


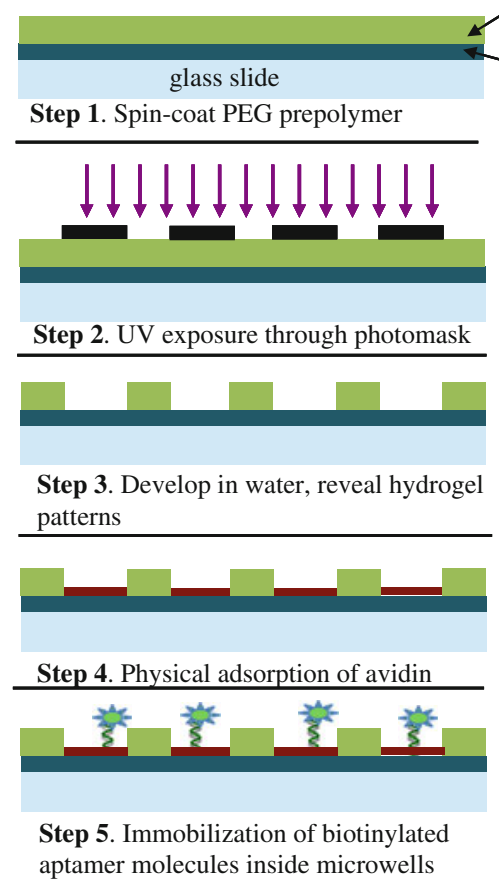

FIGURE 2. Process flow diagram for micropatterning hydrogel microwells and for immobilizing beacon molecules inside the microwells.

introduce acrylate groups on the surface, then covered with liquid prepolymer and exposed to UV through a photomask. This process resulted in micropatterns of cross-linked hydrogel regions interspersed with glass domains. While a variety of hydrogel shapes and geometries can be fabricated using this method, ${ }^{19}$ we chose to work with $35 \mu \mathrm{m}$ diameter microwells since this size approximates dimensions of single cells (5-20 $\mu \mathrm{m}$ diameters) and our intent is to employ sensing microwells for cell analysis in the future. A brightfield image of an array of microwells is shown in Fig. 3a. As a precursor to immobilizing beacon components, the microwells were incubated with avidin. To visualize adsorption, avidin molecules were labeled with Alex546 (red fluorescence). As shown in Fig. 3b, avidin molecules became physically adsorbed on glass attachment sites at the bottom of the microwells, with minimal adsorption occurring on non-fouling hydrogel walls. In a subsequent step, glass substrates were incubated with biotinylated aptamer-Alexa488 (green fluorescence). As demonstrated by a representative image in Fig. 3c, aptamer molecules became immobilized specifically inside the microwells.

\section{Construction of Aptamer Beacons Inside Hydrogel Microwells}

The design of the aptamer beacon is shown in Fig. 1. This beacon consisted of a biotinylated fluorophorelabeled DNA aptamer and a quencher-carrying
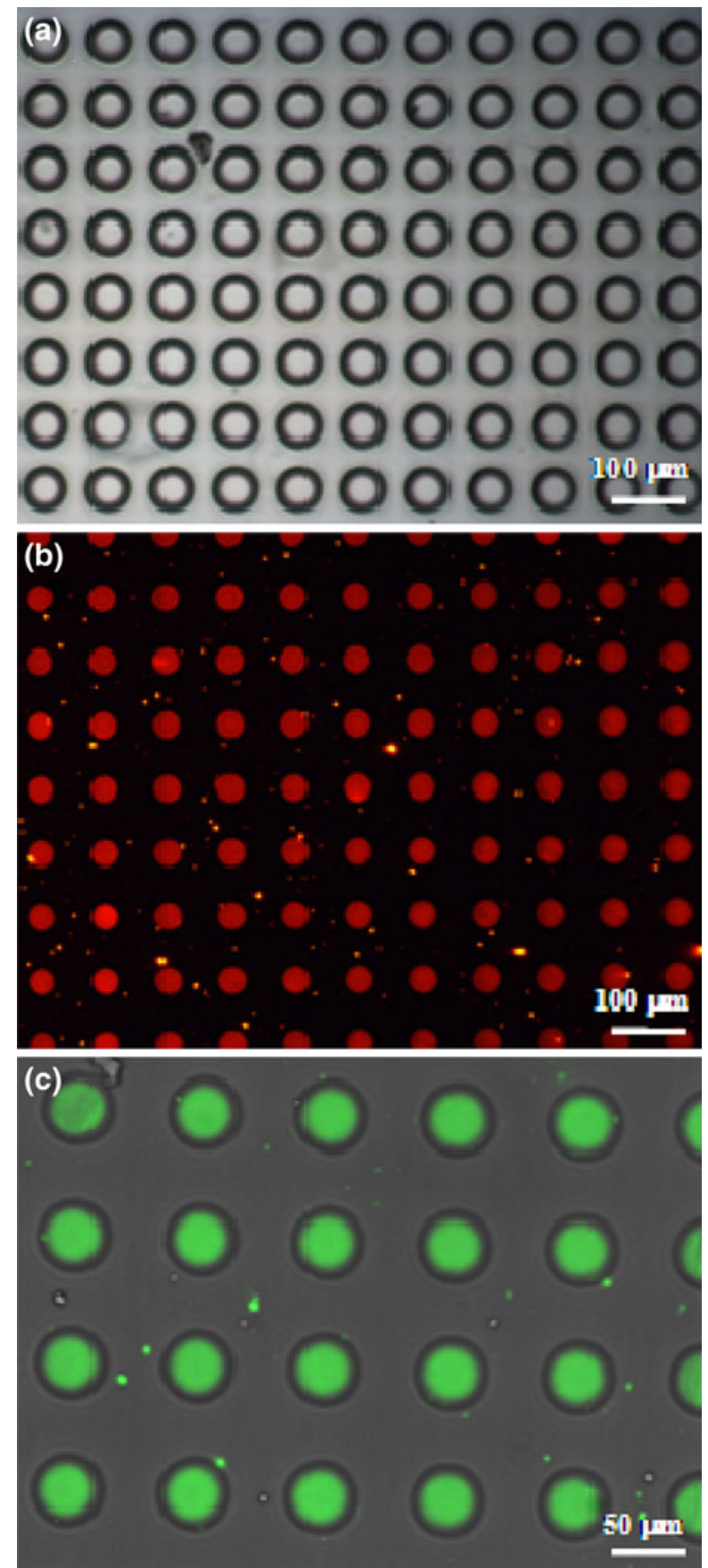

FIGURE 3. Arrays of hydrogel microwells fabricated on glass. (a) A brightfield image showing an array comprised of $35 \mu \mathrm{m}$ diameter wells. (b) An image taken after incubation of a microwell array with avidin conjugated with Alexa546 (red). Localization of fluorescence signal shows that avidin molecules deposited selectively on glass attachment pads inside the wells. Minimal fluorescence was observed on non-fouling PEG hydrogel sidewalls of these wells. (c) Immobilization of biotinylated aptamer conjugated with Alexa488 (green). Aptamer molecules bound selectively inside avidin-containing attachment sites of the microwells.

complementary strand. Hybridization of complementary strand resulted in quenching of fluorescence signal due to FRET effect. As result of IFN- $\gamma$ interacting with 
beacon molecules, quenching strands became displaced and fluorescence signal appeared. SPR analysis carried out in our previous study revealed that best binding $\left(K_{\mathrm{d}} \sim 3 \mathrm{nM}\right)$ occurred in aptamer molecules biotinylated at the $3^{\prime}$-end and that the presence of fluorophore did not significantly interfere with aptamer/IFN- $\gamma$ interactions. ${ }^{28}$

In order to precisely control reagent exchange during IFN- $\gamma$ detection experiments, glass slides with hydrogel microwells were outfitted with PDMS microfluidic channels and mounted on a fluorescence microscope. The design of the microfluidic device, first proposed by Schaff et al..$^{23}$ and shown in Fig. 4a, allowed to reversibly seal PDMS fluid conduits on top of the hydrogel microwell arrays using vacuum suction. This obviated the need to expose micropatterned surfaces to oxygen plasma treatment and allowed to retain intact nonfouling and protein-modified domains on the surface.

Figure 4 demonstrates the last step in construction of the aptamer beacon-attachment of quenching strand and formation of DNA duplex. Figure $4 \mathrm{~b}$ shows that fluorophore-labeled IFN- $\gamma$ aptamer was immobilized via biotin-avidin binding exclusively inside the microwells. Injecting $10 \mu \mathrm{M}$ concentration of the antisense DNA strand labeled with a quencher into the microfluidic device led to disappearance of fluorescence, consistent with FRET-based quenching. As shown in Fig. $4 \mathrm{c}$ and $\mathrm{d}$, quenching occurred rapidly with fluorescence decreasing by $\sim 5$ fold within $20 \mathrm{~s}$, however, aptamer-modified microwells were incubated with the quenching strand for $\sim 30 \mathrm{~min}$ to allow for hybridization to occur. The possibility that the loss of fluorescence was only due to quenching strand being present in solution was eliminated by flushing the device with HKE buffer subsequent to the hybridization step. No fluorescence recovery was observed, suggesting that quenching strands bound to the aptamer molecules and formed double stranded DNA beacons inside the microwells.

\section{Real-time Monitoring of IFN- $\gamma$ in Sensing Microwells}

IFN- $\gamma$ is an important inflammatory cytokine. It is secreted by the immune cells into serum and is most commonly detected using antibody (Ab)-based affinity assays. In immunology, a standard ELISA has evolved into an ELISpot assay - a method used to detect IFN- $\gamma$ secreted by T-cells and to determine frequency of antigen-specific CD4 and CD8 T-cells. ${ }^{3,5}$ While enumeration of IFN- $\gamma$ producing immune cells has become standard in immunology, the quantity and the dynamics of cytokine production in cells are largely unknown. The latter point is particularly difficult to address given that $\mathrm{Ab}$-based assays provide end-point measurements and offer limited information on the cytokine production over time.
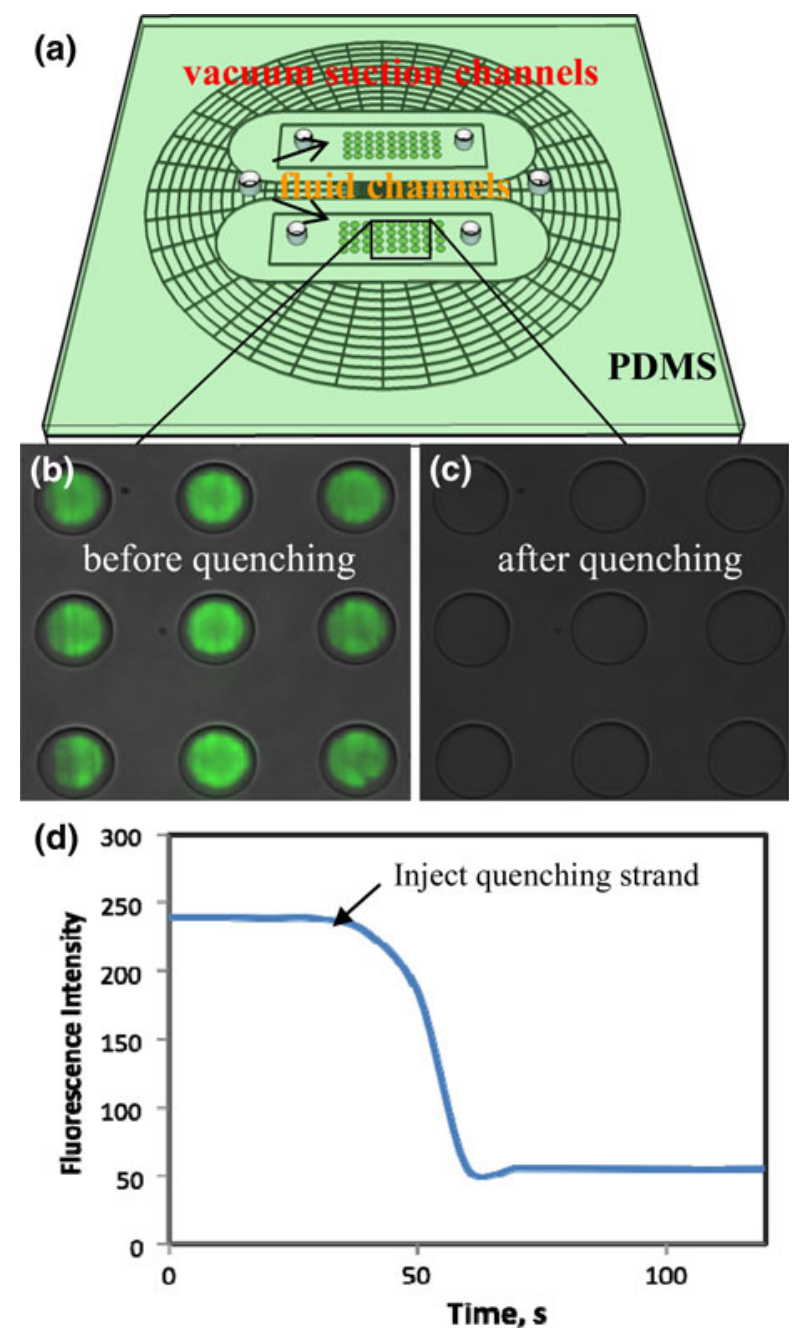

FIGURE 4. (a) Design of a microfluidic device used in cytokine detection studies. The device was fabricated in PDMS and contained two reaction chambers that were secured on top of microwell arrays by vacuum suction applied through a web of auxiliary channels. This PDMS attachment approach did not compromise non-fouling or sensing components of micropatterned surfaces. (b-c) $35 \mu \mathrm{m}$ diameter wells with immobilized aptamer molecules before (b) and after (c) injection of quencher-labeled complementary strands into a microfluidic device. Loss of fluorescence in part (c) was attributed to formation of DNA duplex. (d) Fluorescence intensity measurements show 5 fold decrease in fluorescence after incubation of microwells with quenching DNA strands.

Micropatterning of aptamer beacons on cell culture/ capture surfaces present an alternative strategy for monitoring cytokine signal in real-time. Figure 5a-c shows a representative response of hydrogel microwells to $100 \mathrm{nM}$ IFN- $\gamma$ injected into the microfluidic device. As can be seen from these images fluorescence signal inside the microwells increased rapidly, reaching saturation $\sim 15 \mathrm{~s}$ after introduction of IFN- $\gamma$. Similar dynamics for IFN- $\gamma /$ aptamer beacon interactions have been observed by us previously using SPR analysis. ${ }^{28}$ The appearance of fluorescence in Fig. $5 \mathrm{a}-\mathrm{c}$ is due to 


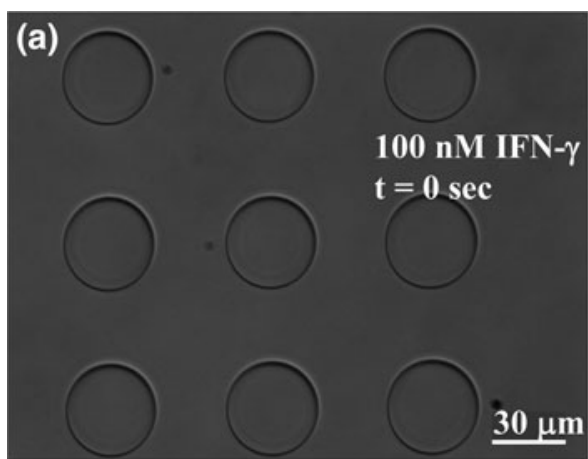

(d)

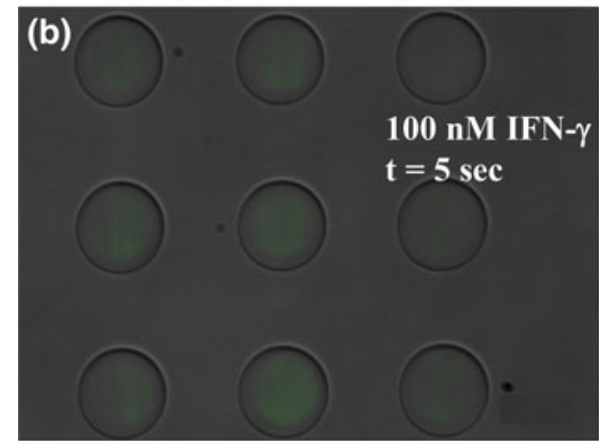

(e)
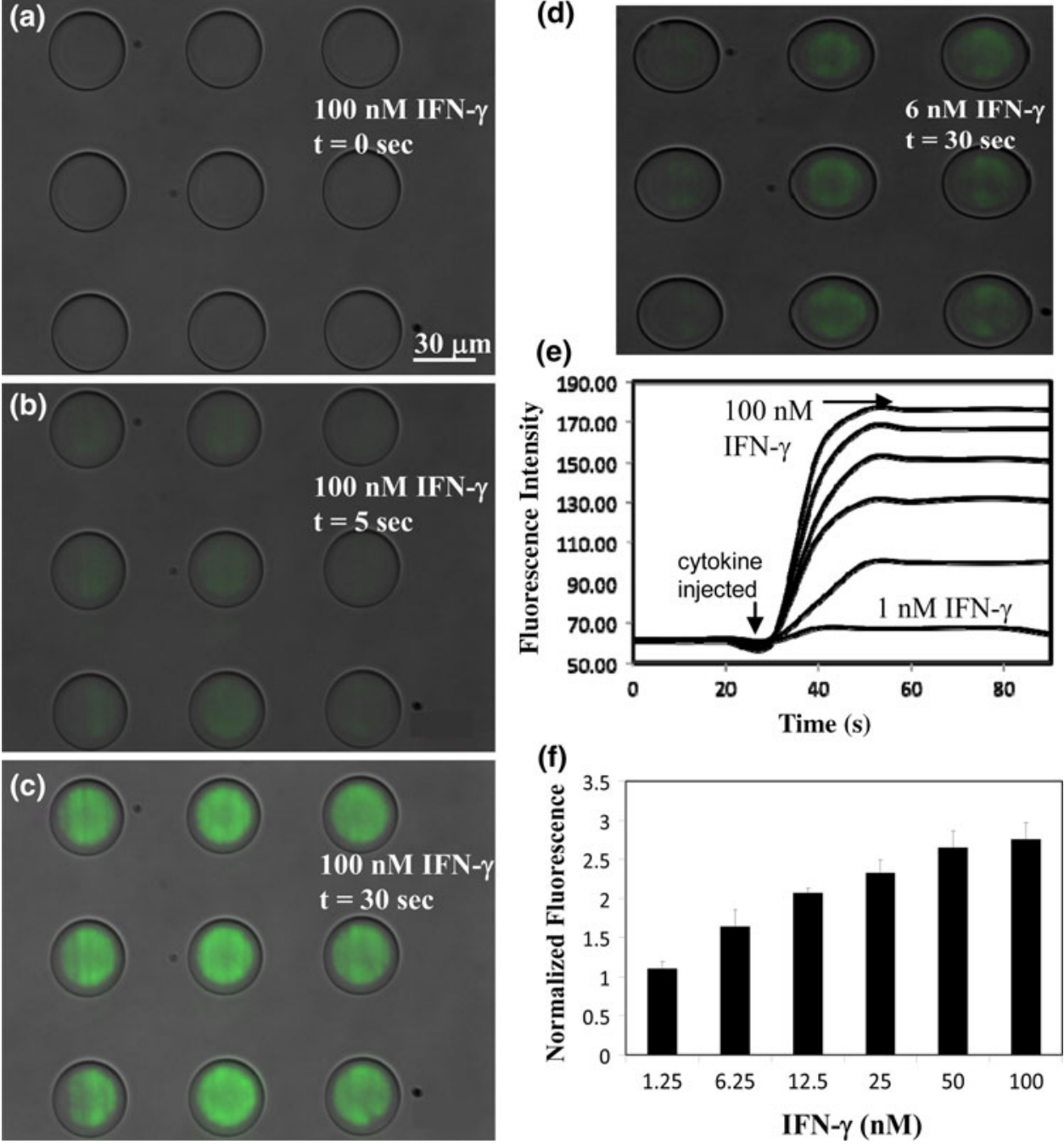

FIGURE 5. (a-c) A sequence of fluorescence/brightfield overlay images taken at different time points from a set of microwells challenged with $100 \mathrm{nM}$ IFN- $\gamma$. Appearance of fluorescence in the microwells is due to displacement of quenching strands in the immobilized beacons by quenching strands. (d) Fluorescence response of a different set of microwells challenged with $6 \mathrm{nM}$ IFN- $\gamma$. The signal was considerably lower than in the case of microwells challenged with $100 \mathrm{nM}$ IFN- $\gamma$ (c), proving that microwell response depended on the concentration of analyte. (e) Temporal evolution of fluorescence signal in microwells challenged with varying concentrations of IFN- $\gamma$. (f) A plot of signal intensity vs. IFN- $\gamma$ concentration showing that detection limit for these sensing surfaces was $5 \mathrm{nM}$ and linear range extended to $100 \mathrm{nM}$. Error bars represent standard deviation of the mean for three $(n=3)$ separate experiments. The values for fluorescence intensity were chosen from curves in part (e) after signal saturation (e.g. $t=60 \mathrm{~s}$ ). Fluorescence signal was normalized by background fluorescence $\left(F_{0}\right)$.

displacement of the quenching strand by IFN- $\gamma$ molecules and disruption of FRET. As seen from these images signal was localized to microwells, underscoring our ability to define cytokine-sensing domains on the glass surface.

It should be noted that IFN- $\gamma(17 \mathrm{kDa})$ is slightly bigger than the aptamer $(10 \mathrm{kDa})$, which means that controlling aptamer density on the surface is important in order to ensure binding of cytokine molecules. In the previous study employing electrochemical IFN- $\gamma$ aptamer we determined that modifying surfaces with $0.5-1 \mu \mathrm{M}$ aptamer solution resulted in most sensitive
IFN- $\gamma$ detection. ${ }^{12}$ Similar aptamer concentration $(1 \mu \mathrm{M})$ was employed in the present study for fabrication of cytokine sensing surfaces.

Comparison of fluorescence in microwells challenged with $100 \mathrm{nM}$ (Fig. 5c) and $6 \mathrm{nM} \mathrm{IFN- \gamma} \mathrm{(Fig.} \mathrm{5d)} \mathrm{dem-}$ onstrates that signal intensity was dependent on analyte concentration. Figure 5e presents dynamics of signal change for varying IFN- $\gamma$ concentrations (from 1 to 100 $\mathrm{nM}$ ) and points to equilibrium being achieved rapidly ( $\sim 15 \mathrm{~s}$ after introduction of analyte). The fluorescence intensity at equilibrium varied depending on analyte concentration, allowing us to construct a calibration 
curve of signal vs. IFN- $\gamma$ concentration. As seen from these data (Fig. 5f), the detection limit of micropatterned surfaces is $5 \mathrm{nM}$, with linear range extending to $100 \mathrm{nM}$ IFN- $\gamma$. Fitting results in Fig. 5e to Langmuir isotherm provided an equilibrium constant $K_{\mathrm{d}}$ of $\sim 2.5 \mathrm{nM}$ for IFN- $\gamma /$ aptamer binding. This value was similar to $K_{\mathrm{d}}$ of $3 \mathrm{nM}$ measured by us previously using $\mathrm{SPR}^{28}$

The micropatterned surfaces described here are not as sensitive as Ab-based IFN- $\gamma$ assays. However, our laboratory has recently reported an electrochemical aptasensor with $60 \mathrm{pM}$ detection limit. ${ }^{12}$ This electrochemical biosensor used a hairpin aptamer that showed significantly higher affinity for IFN- $\gamma$ compared to linear aptamer described here (hairpin $K_{\mathrm{d}}-0.3 \mathrm{nM}$, linear aptamer $K_{\mathrm{d}}-3 \mathrm{nM}$ determined by SPR). Aptamer beacons utilizing hairpin configuration are currently under development in our laboratory and may provide a more sensitive fluorescence-based detection of IFN- $\gamma$.

In addition to real-time analyte monitoring, aptamers offer an advantage of chemical stability. The lack of chemical stability makes storage of Ab-based assays difficult and re-use of these assays nearly impossible. In contrast, as shown in Fig. 6, aptamer-based biosensing surfaces could be reused multiple times with minimal loss in sensitivity. In these experiments, microwells with immobilized aptamer beacon (DNA duplex) were challenged with $100 \mathrm{nM} \mathrm{IFN- \gamma}$ as described in the previous section of this paper. Subsequently, microfluidic devices were infused with $7 \mathrm{M}$ urea buffer for $5 \mathrm{~min}$. This treatment was sufficient to disrupt aptamer-cytokine interactions and regenerate fluorescence of sensing

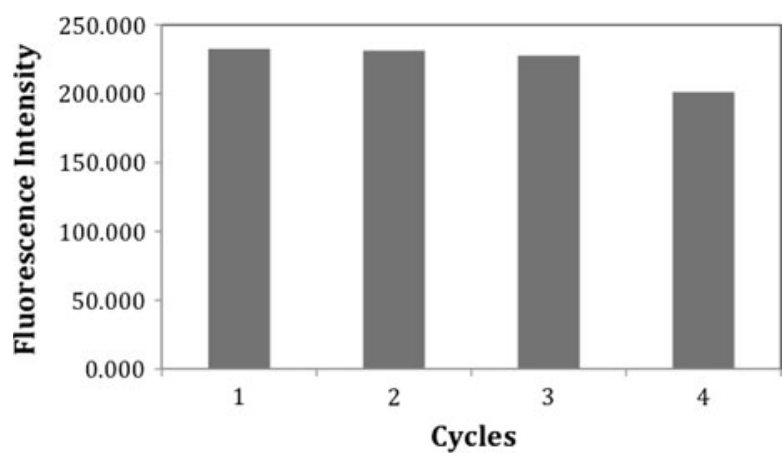

FIGURE 6. Regeneration of cytokine-sensing surfaces. Hydrogel microwells containing aptamer beacons (DNA duplex) were exposed to $100 \mathrm{nM}$ IFN- $\gamma$, producing fluorescence signal. Subsequently, microwells were washed with urea buffer to denature/remove IFN- $\gamma$ and regenerate fluorescence of the sensing surface. In the last step, hydrogel microwells were incubated with the quenching strand to regenerate aptamer beacon (DNA duplex). This process allowed us to use the same sensing microwells multiple times with minimal loss of sensitivity. surfaces. As can be seen from Fig. 6, the same micropatterned surface regenerated and challenged with $100 \mathrm{nM}$ IFN- $\gamma$ showed minimal cycle-to-cycle variability.

\section{CONCLUSIONS}

Micropatterned surfaces may represent a future of high-throughput screening because of the high-density of wells per unit area afforded by microfabrication technologies. A number of reports described the use of micropatterned surfaces to arrange cells into periodic arrays suitable for rapid imaging and characterization. ${ }^{4,18,21,31}$ However, options for monitoring function of live cells on micropatterned surfaces remain limited, with reporter genes ${ }^{10,31}$ and intracellular fluorophores ${ }^{22}$ being used most commonly. This paper describes fabrication of microwell arrays suitable for real-time monitoring of IFN- $\gamma$ - an important protein secreted by immune cells during activation. PEG hydrogel microwells ( $35 \mu \mathrm{m}$ diameter) were fabricated on glass using photolithography and were modified with aptamer beacon molecules. Hydrogel walls remained non-fouling so that beacon molecules became localized and immobilized on glass attachment sites inside the microwells. Microwells fabricated in this manner emitted fluorescence upon incubation with IFN- $\gamma$. The fluorescence signal intensity changed in real-time and was dependent on the concentration of IFN- $\gamma$.

Our laboratory has recently demonstrated fabrication of microwells modified with anti-CD4 and anti-IFN- $\gamma$ Abs for the capture of single human CD4 T-cells from whole blood and detection of secreted IFN $-\gamma^{33}$ As the next step, we envision replacing IFN- $\gamma$ Abs with aptamer beacons to allow capturing T-cells and monitoring dynamics of IFN- $\gamma$ release from these cells. These micropatterned surfaces may enable analysis of heterogeneity in immune cell population based on the dynamics of cytokine production. Overall, micropatterning of aptamer beacons on cell culture/capture surfaces represents a novel means of monitoring cell-secreted products in the context of local microenvironment.

\section{ACKNOWLEDGMENTS}

The authors would like to acknowledge Prof. Yohei Yokobayashi at UC Davis for helpful advice regarding aptamer beacon construction. The use of fluorescence microscopy equipment in the laboratory of Prof. Laura Marcu at UC Davis is greatly appreciated. We thank Dr. Michael Howland and Ms. Jun Yan for help with manuscript preparation. This work was supported by an NSF EFRI (\#0937997) grant awarded to AR. 


\section{OPEN ACCESS}

This article is distributed under the terms of the Creative Commons Attribution Noncommercial License which permits any noncommercial use, distribution, and reproduction in any medium, provided the original author(s) and source are credited.

\section{REFERENCES}

${ }^{1}$ An, W. F., and N. Tolliday. Cell-based assays for highthroughput screening. Mol. Biotechnol.45(2):180-186, 2010.

${ }^{2}$ Bradshaw, E. M., S. C. Kent, V. Tripuraneni, T. Orban, H. L. Ploegh, D. A. Hafler, and J. C. Love. Concurrent detection of secreted products from human lymphocytes by microengraving: Cytokines and antigen-reactive antibodies. Clin. Immunol. 129(1):10-18, 2008.

${ }^{3}$ Britten, C. M., R. G. Meyer, T. Kreer, I. Drexler, T. Wolfel, and W. Herr. The use of HLA-A0201-transfected K562 as standard antigen-presenting cells for C8 T lymphocytes in IFN-gamma ELISPOT assays. J. Immun. Methods 259:95-110, 2002.

${ }^{4}$ Charnley, M., M. Textor, A. Khademhosseini, and M. P. Lutolf. Integration column: microwell arrays for mammalian cell culture. Integr. Biol. 1(11-12):625-634, 2009.

${ }^{5}$ Cox, J. H., G. Ferrari, and S. Janetzki. Measurement of cytokine release at the single cell level using ELISPOT assay. Methods 38:274-282, 2006.

${ }^{6}$ El-Ali, J., P. K. Sorger, and K. F. Jensen. Cells on chips. Nature 442:403-411, 2006.

${ }^{7}$ Ellington, A. D., and J. W. Szostak. Invitro selection of RNA molecules that bind specific ligands. Nature 346(6287):818-822, 1990

${ }^{8}$ Hamaguichi, N., A. D. Ellington, and M. Stanton. Aptamer beacons for the direct detection of proteins. Anal. Biochem. 294:126-131, 2001.

${ }^{9}$ Han, Q., E. M. Bradshaw, B. Nilsson, D. A. Hafler, and J. C. Love. Multidimensional analysis of the frequencies and rates of cytokine secretion from single cells by quantitative microengraving. Lab Chip 10(11):1391-1400, 2010.

${ }^{10}$ King, K. R., S. H. Wang, D. Irimia, A. Jayaraman, M. Toner, and M. L. Yarmush. A high-throughput microfluidic real-time gene expression living cell array. Lab Chip 7(1):77-85, 2007.

${ }^{11} \mathrm{Li}$, J. J., X. Fang, and W. Tan. Molecular aptamer beacons for real-time protein recognition. Biochem. Biophys. Res. Commun. 292:31-40, 2002.

${ }^{12}$ Liu, Y., N. Tuleuova, E. Ramanculov, and A. Revzin. Aptamer-based electrochemical biosensor for interferon gamma detection. Anal. Chem. doi:10.1021/ac101409t2010.

${ }^{13}$ Michelini, E., L. Cevenini, L. Mezzanotte, A. Coppa, and A. Roda. Cell-based assays: fuelling drug discovery. Anal. Bioanal. Chem. 398(1):227-238, 2010.

${ }^{14}$ Minor, L. K. Label-free cell-based functional assays. Comb. Chem. High Throughput Screen. 11(7):573-580, 2008.

${ }^{15}$ Norris, P. J., and E. S. Rosenberg. CD4 T helper cells and the role they play in viral control. J. Mol. Med. 80:397-405, 2002.

${ }^{16}$ Pantaleo, G., and A. Harari. Functional signature in antiviral T-cell immunity for monitoring virus-associated diseases. Nat. Rev. Immun. 6:417-423, 2006.
${ }^{17}$ Pantaleo, G., and R. A. Koup. Correlates of immune protection in HIV-1 infection: what we know, what we do not know, what we should know. Nat. Med. 10:806-810, 2004.

${ }^{18}$ Rettig, J. R., and A. Folch. Large-scale single-cell trapping and imaging using microwell arrays. Anal. Chem. 77(17): 5628-5634, 2005.

${ }^{19}$ Revzin, A., R. J. Russell, V. K. Yadavalli, W.-G. Koh, C. Deister, D. D. Hile, M. B. Mellott, and M. V. Pishko. Fabrication of poly(ehtylene glycol) hydrogel microstructures using photolithography. Langmuir 17:5440-5447, 2001.

${ }^{20}$ Revzin, A., R. G. Tompkins, and M. Toner. Surface engineering with poly(ethylene glycol) photolithography to create high-density cell arrays on glass. Langmuir 19: 9855-9862, 2003.

${ }^{21}$ Revzin, A., K. Sekine, A. Sin, R. G. Tompkins, and M. Toner. Development of a microfabricated cytometry platform for characterization and sorting of individual leukocytes. Lab Chip 5:30-37, 2005.

${ }^{22}$ Roach, K. L., K. R. King, K. Uygun, S. C. Hand, I. S. Kohane, M. L. Yarmush, and M. Toner. Highthroughput single cell arrays as a novel tool in biopreservation. Cryobiology 58(3):315-321, 2009.

${ }^{23}$ Schaff, U. Y., M. M. Q. Xing, K. K. Lin, N. Pan, N. L. Jeon, and S. I. Simon. Vascular mimetics based on microfluidics for imaging the leukocyte-endothelial inflammatory response. Lab Chip 7(4):448-456, 2007.

${ }^{24}$ Story, C. M., E. Papa, C. C. A. Hu, J. L. Ronan, K. Herlihy, H. L. Ploegh, and J. C. Love. Profiling antibody responses by multiparametric analysis of primary B cells. Proc. Nat. Acad. Sci. USA 105(46):17902-17907, 2008.

${ }^{25}$ Stybayeva, G., O. Mudanyali, S. Seo, J. Silangcruz, M. Macal, E. Ramanculov, S. Dandekar, A. Erlinger, A. Ozcan, and A. Revzin. Lensfree holographic imaging of antibody microarrays for high-throughput detection of leukocyte numbers and function. Anal. Chem. 82(9): 3736-3744, 2010.

${ }^{26}$ Tan, W. H., K. M. Wang, and T. J. Drake. Molecular beacons. Curr. Opin. Chem. Biol. 8(5):547-553, 2004.

${ }^{27}$ Thomas, C. H., J. H. Collier, C. S. Sfeir, and K. E. Healy. Engineering gene expression and protein synthesis by modulation of nuclear shape. Proc. Nat. Acad. Sci. USA 4:1972-1977, 2002.

${ }^{28}$ Tuleuova, N., C. N. Jones, J. Yan, E. Ramanculov, Y. Yokobayashi, and A. Revzin. Development of an aptamer beacon for detection of interferon-gamma. Anal. Chem. 82(5):1851-1857, 2010.

${ }^{29}$ Wlodkowic, D., and J. M. Cooper. Microfabricated analytical systems for integrated cancer cytomics. Anal. Bioanal. Chem. 398(1):193-209, 2010.

${ }^{30}$ Wolcke, J., and D. Ullmann. Miniaturized HTS technologies-uHTS. Drug Discov. Today 6:637-646, 2001.

${ }^{31}$ Yarmush, M. L., and K. R. King. Living-cell microarrays. Annu. Rev. Biomed. Eng. 11:235-257, 2009.

${ }^{32}$ Zhu, H., G. S. Stybayeva, M. Macal, M. D. George, S. Dandekar, and A. Revzin. A microdevice for multiplexed detection of T-cell secreted cytokines. Lab Chip 8:2197-2205, 2008.

${ }^{33}$ Zhu, H., G. S. Stybayeva, J. Silangcruz, J. Yan, E. Ramanculov, S. Dandekar, M. D. George, and A. Revzin. Detecting cytokine release from single human T-cells. Anal. Chem. 81:8150-8156, 2009. 